\title{
Minimum Energy Multicast Routing in Mobile Ad-Hoc Network
}

\author{
Mrunali S.Sonwalkar \\ Dept.of Computer science and engg. \\ M.B.E.S.College of engineering. \\ Ambajogai. India
}

\author{
Rammana S.Havinal \\ Dept.of Computer science and engg. \\ M.B.E.S.College of engineering. \\ Ambajogai. India
}

\begin{abstract}
A Mobile Ad-hoc Network (MANET) is a collection of mobile nodes. It can be constructed anywhere without any infrastructure. The mobile nodes are equipped with energylimited batteries. An important issue in such a network is to minimize the total energy consumption for each operation. In MANET each node acts as a store and forward station for routing packets. As the nodes are highly dynamic, maintaining routes become a greater challenge.

Multicasting is one of the fundamental mechanism, which can be typically implemented by creating a multicast tree. It is achieved by forming minimum spanning tree between the source nodes and other mobile nodes in the network and then data is transmitted over this minimum path. In this paper we are focusing on designing energy efficient routing algorithms for creating minimum spanning tree. It first uses AODV as basic protocol, then we are designing a distributed algorithm for finding the approximate distance between nodes. We have conducted experiments by simulations to evaluate the performance of the proposed algorithm and compare it with the basic AODV. The experimental results demonstrate that the proposed algorithm significantly reduces energy consumption, delay and improves throughput and packet delivery ratio.
\end{abstract}

\section{Keywords}

MANET, energy conservation, delay, throughput, multicasting, minimum spanning tree, distributed algorithm.

\section{INTRODUCTION}

A Mobile Ad-hoc Network (MANET) is a collection of mobile nodes, equipped with wireless communication devices.

These wireless communication devices are connected by wireless links without any central infrastructure. These networks introduced a new art of network establishment and can be well suited for an environment where either the infrastructure is lost or establishing an infrastructure is very cost effective.

Each device in a MANET is free to move independently in any direction, and therefore change its links to other devices frequently. Each device must forward traffic unrelated to its own use, and therefore be a router. The primary challenge in building a MANET is equipping each device to continuously maintain the information required to properly route traffic. Such networks may operate by themselves or may be connected to the larger Internet.

Each mobile node is operated by a energy limited battery and usually it is impossible to recharge or replace the batteries during a operation. However, the set of network links between the mobile nodes and their capacities is not predetermined because it depends on factors such as distance between nodes, transmission power, hardware implementation and environmental noise [1]. The communication between two mobile nodes can be either in a single hop transmission in

which case the two nodes are within the transmission ranges of each other, or in a multi-hop transmission where the message is relayed by intermediate mobile nodes. It is well known that wireless communications consume significant amounts of battery power therefore; the limited battery lifetime imposes a severe constraint on the network performance. Energy conservation in such a network thus is of paramount importance, and energy efficient operations are critical to prolong the lifetime of the network.

Energy conservation techniques for ad hoc networks can be broadly classified into two categories: power mode control and transmission power control. A power mode control protocol aims to put wireless nodes into periodical sleep state in order to reduce the power consumption in the idle listening mode. Transmission power control manages energy consumption by adjusting transmission ranges during actual transmission [2].

The fundamental problem in mobile ad hoc network is energy management and one of the solutions to this problem is multicasting. Multicasting plays a crucial role in MANETs to support its number of applications. It is an efficient mechanism for one to many communications, and is typically implemented by creating a multicast tree. It involves the transmission of a packet to a group of zero or more hosts identified by a single destination address, and so is intended for group-oriented computing. A multicast packet is delivered to all members of its destination host group.

The use of multicasting within MANETs has many benefits. It can reduce the cost of communication and improve the efficiency of the wireless channel when sending multiple copies of the same data by exploiting the inherent broadcasting properties of wireless transmission.

Due to limited battery power and transmission bandwidth limitations, in wireless ad hoc networks, it is essential to develop efficient multicast protocols that are optimized for energy consumption and significantly improving networks overall performance. Multicasting also involves an all-to-all multicast session consisting of a set of terminal nodes in an ad hoc network, where the transmission power of each node is either fixed or adjustable. The set of network nodes which may generate a multicast packet to be distributed to a multicast group are referred to as source nodes[3].

Multicasting is achieved by forming minimum spanning tree between these source nodes and other mobile nodes in the 
network and then data is transmitted over this minimum path which in turn manages the energy associated with the nodes.

\section{RELATED WORK}

A major concern in mobile ad hoc networks (MANETs) is energy conservation. It is due to the limited lifetime of batteries. A great effort has been devoted to develop energyaware network protocols .V.Ramesh proposed in [2] a new communication mechanism called RandomCast, which is a message forwarding mechanism related with the energy and the overall network performance. In RandomCast, a node may decide not to overhear (a unicast message) and not to forward (a broadcast message) when it receives an advertisement during an ATM window, thereby reducing the energy cost without deteriorating the network performance. It is highly energy-efficient compared to conventional 802.11 schemes, in terms of total energy consumption, energy good-put, and energy balance.

Mobile ad-hoc networking involves peer-to-peer communication in a network with a dynamically changing topology. Weifa Liang proposed in [3] a symmetric wireless ad hoc network. In this network, the minimum-energy multicast tree is devised by considering the number of approximations according to the total length of network. The approximation algorithm analyses power at every node $v_{i}$ i.e. $\mathrm{p}_{\mathrm{wi}}$. Then the range of the battery power at node $\mathrm{v}_{\mathrm{i}}$ is partitioned into a number of power intervals and each of them corresponds to a power level.It assumes, the power interval of each corresponding power node is in the range of $2^{p 1}<p w i<2^{p i+1}$. Based on these power intervals the approximation solution calculates the optimum value with which the power associated with every node is within a constant factor and thus it is minimum.

Another solution proposed in [4] by Weifa Liang et.al, uses, the minimum-energy multicasting approach, that can be used by employing one-to-many communication mechanism. That is, an energy efficient multicast tree is rooted at each terminal node. One node acts as a source node from which a single multicast tree is constructed which is shared by other nodes to multicast its messages to the remaining terminal nodes. It also considers an all to- all multicast session with a terminal set $\mathrm{D}$, such that each terminal node $\mathrm{v} \in \mathrm{D} \subseteq \mathrm{N}$ has a message of length $l_{\mathrm{v}}$ to share with the others in $\mathrm{D}$, the minimum-energy all to- all multicasting problem is to construct a shared multicast tree spanning the nodes in $\mathrm{D}$ such that the total energy consumption of realizing the all-to-all multicast session using the tree is minimized.

In ad-hoc network the nodes are mobile and their topologies are dynamically changing, therefore it becomes difficult to built a minimum spanning tree. Yongwook Choi, \& Maleq Khan proposed in [5] the minimum spanning tree (MST) problem, by assuming random distribution of nodes over the Euclidean distance of the network. It uses a energy efficient distributed algorithm for Euclidean MST problem. This algorithm calculates the energy complexity by considering its lower bound and upper bound. It then uses its lower bound to construct MST It also uses constant energy algorithm that gives a constant factor approximation to the MST. It then constructs a energy model which focuses over minimizing transmission energy by considering energy complexity.

Hassan Artail and Khaleel Mershad proposed in [6] introduced a message forwarding algorithm that is based on the concept of selecting the nearest node from a set of designated nodes. This algorithm is called as Minimum Distance Packet Forwarding (MDPF). The goal of the proposed algorithm is to minimize the average number of hops taken to reach the node that holds the desired data. Numerical analysis and experimental evaluations produced by the algorithm also helps to derive the lower and upper bounds of the interval for the hop count. It also decides the mean hop count between the source node and the destination node. In the experimental evaluation, the performance of MDPF was compared with Random Packet Forwarding (RPF) and Minimal Spanning Tree Forwarding (MSTF). With the help of results produced by the numerical analysis, the author stated that the MDPF offers significant hop count savings and smaller delays when compared to RPF and MSTF.

Maleq Khan and Gopal Pandurangan proposed in [7] a class of simple and local distributed algorithms called Nearest Neighbor Tree (NNT) algorithms for energy-efficient construction of an approximate MST in wireless networks. It generates NNT algorithms for the complete graph model. Depending on the ranks of the nodes, it generates two NNT algorithms: Random-NNT (ranks are chosen randomly) and Coordinate-NNT (Co-NNT in short; ranks are based on coordinates of the nodes). The main results derived from algorithm are: (i) The tree produced by such an algorithm, called the NNT, has low cost, (ii) The NNT paradigm can be used to design a simple dynamic algorithm for maintaining a low cost spanning tree, and (iii) The time, message and work complexities of the NNT algorithms are close to the optimal in several settings.

In ad-hoc network the nodes are mobile and their topologies are dynamically changing, therefore it becomes difficult to built a minimum spanning tree. Yongwook Choi, \& Maleq Khan proposed in [5] the minimum spanning tree (MST) problem, by assuming random distribution of nodes over the Euclidean distance of the network. It uses a energy efficient distributed algorithm for Euclidean MST problem. This algorithm calculates the energy complexity by considering its lower bound and upper bound. It then uses its lower bound to construct MST It also uses constant energy algorithm that gives a constant factor approximation to the MST. It then constructs a energy model which focuses over minimizing transmission energy by considering energy complexity.

Hassan Artail and Khaleel Mershad proposed in [6] introduced a message forwarding algorithm that is based on the concept of selecting the nearest node from a set of designated nodes. This algorithm is called as Minimum Distance Packet Forwarding (MDPF). The goal of the proposed algorithm is to minimize the average number of hops taken to reach the node that holds the desired data. Numerical analysis and experimental evaluations produced by the algorithm also helps to derive the lower and upper bounds of the interval for the hop count. It also decides the mean hop count between the source node and the destination node. In the experimental evaluation, the performance of MDPF was compared with Random Packet Forwarding (RPF) and Minimal Spanning Tree Forwarding (MSTF). With the help of results produced by the numerical analysis, the author stated that the MDPF offers significant hop count savings and smaller delays when compared to RPF and MSTF.

Maleq Khan and Gopal Pandurangan proposed in [7] a class of simple and local distributed algorithms called Nearest Neighbor Tree (NNT) algorithms for energy-efficient construction of an approximate MST in wireless networks. It generates NNT algorithms for the complete graph model. 
Depending on the ranks of the nodes, it generates two NNT algorithms: Random-NNT (ranks are chosen randomly) and Coordinate-NNT (Co-NNT in short; ranks are based on coordinates of the nodes). The main results derived from algorithm are: (i) The tree produced by such an algorithm, called the NNT, has low cost, (ii) The NNT paradigm can be used to design a simple dynamic algorithm for maintaining a low cost spanning tree, and (iii) The time, message and work complexities of the NNT algorithms are close to the optimal in several settings.

\section{PROPOSED SYSTEM}

\subsection{Wireless communication model}

A wireless ad hoc network can be modeled by an undirected graph $\mathrm{M}=(\mathrm{N}, \mathrm{A})$, where $\mathrm{N}$ is the set of homogeneous stationary nodes and $\mathrm{A}$ is the set of links with $\mathrm{n}=|\mathrm{N}|$ and $\mathrm{m}=$ $|A|$. Each multicast request is a pair $(S, D)$ where $s$ is the source node and D is the set of destination nodes. There is an edge $(\mathrm{u}, \mathrm{v}) € \mathrm{~A}$ if nodes $\mathrm{u}$ and $\mathrm{v}$ are within the transmission ranges of each other. For any edge $(\mathrm{u}, \mathrm{v}) € \mathrm{~A}$, its two endpoints $\mathrm{u}$ and $\mathrm{v}$ are called neighboring nodes[4].

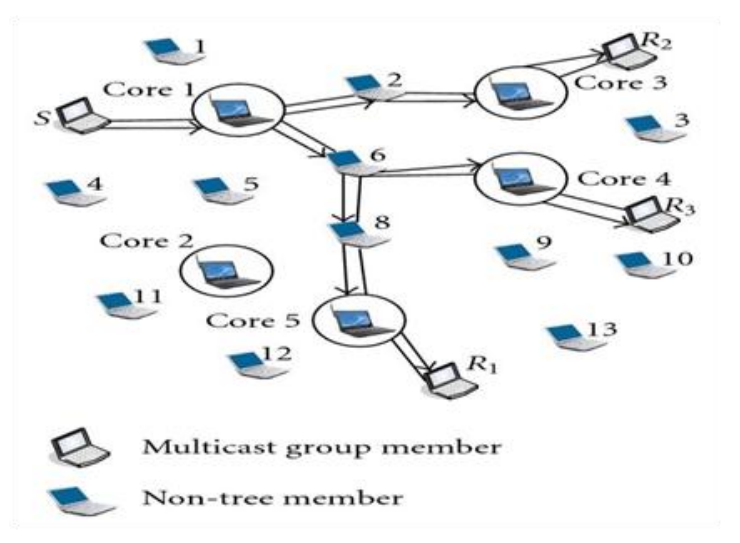

Fig 1: Multicast data forwarding[1].

We assume that the network topology is stable during the processing period of a multicast request, where we say processing a multicast request, means that the system either builds a multicast tree for and realizes the request using the built tree, or rejects the request if there are not enough network resources to accommodate the request[8]. Typical multicast data forwarding is as shown in Fig 1. After it has finished processing the current multicast request and before its response to the next multicast request, the system allows the nodes in the network to move and a new network topology is then formed. Each node in the network is equipped with omnidirectional antenna and the transmission power at the node is finitely or infinitely adjustable[9].

Each node can choose one of its power levels to transmit messages. In other words, we assume that there are $l_{i}$ power levels at node $\mathrm{v}_{\mathrm{i}} € \mathrm{~N}$. Let $\mathrm{w}_{\mathrm{i}}$ be the power of vi at its power level 1 .Among the $1_{i}$ power levels, one is the minimum operational power level with power $\mathrm{p}_{\min (\mathrm{vi})}$ and another is the maximum operational power level with power $\mathrm{p}_{\max (\mathrm{vi}) \text {, }}$ Furthermore, given two neighboring nodes $u$ and $v$, there is always a corresponding power level between $\mathrm{u}$ and $\mathrm{v}$ with the same amount of power, which we refer to as the power level symmetry of neighboring nodes[11]. Obviously, the amount of power to maintain the power level symmetry between $u$ and $\mathrm{v}$ is the minimum power required to keep them within the transmission range of each other. For a transmission in the network from node $u$ to node $\mathrm{v}$, separated by a distance $\mathrm{d}_{\mathrm{u}, \mathrm{v}}$, to guarantee that $\mathrm{v}$ is within the transmission range of $\mathrm{u}$, the transmission power

at $\mathrm{u}$ is modeled to be proportional to $\mathrm{d}_{\mathrm{u}, \mathrm{v}}^{\alpha}$, assuming that the proportionality constant is 1 for notational simplicity, $\alpha$ is a parameter that typically takes a value between 2 and 4 , depending on the characteristics of the communication medium The reachability of a node in wireless ad hoc networks is fully determined by the transmission power at the node.

\subsection{The minimum energy all-to-all multicasting problem}

Given a wireless ad hoc network M(N,A).A multicast request consisting of a source node $\mathrm{S}$ and a destination set $\mathrm{D}$, the minimum-energy multicast tree problem is to construct a multicast tree rooted at the source node and spanning the nodes in $\mathrm{D}$ such that the sum of transmission power at non leaf nodes is minimized. The multicast tree changes as the source and destination node changes. Before transmitting a message, the source node find out the minimum path over which energy consumption can be reduced, then it transmit the message over that path. To find out new path every time, network uses distributed algorithm to parse the network. After transmitting the message over this path, the remaining energy, consumed energy, packet delivery ratio, end-to-end delay, throughput can be calculated. All these parameters also dependent upon the packet size and data rate.

For constructing multicast session with minimum spanning tree the distributed algorithm is used, which can be of two types (i) For fixed transmission power (ii) For adjustable transmission power. An example of a network and its minimum spanning tree with multicasting is as shown in Fig. 2.

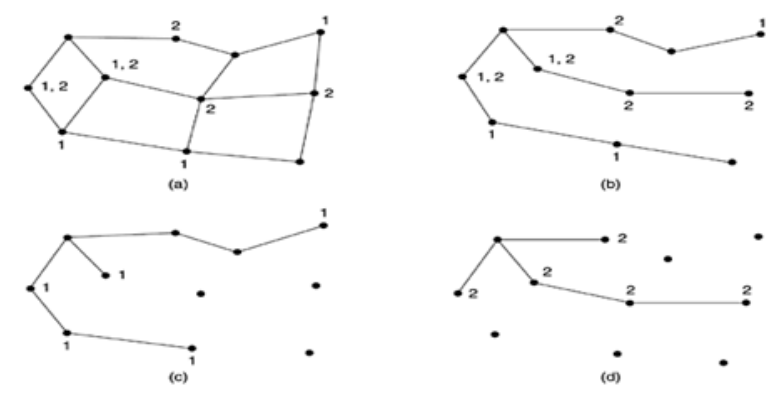

Fig. 2: (a) A network (b) Minimum spanning tree (c) Minimum spanning tree with multicast in group 1(d) Minimum spanning tree with multicast in group 2[1].

\subsection{Distributed algorithm}

Each node has the local knowledge of its neighboring nodes[4]. Based on such a distributed environment, the centralized approximation algorithm can be simplified with its distributed implementation, which is referred to as algorithm Dist_Implement as shown in Fig 3 For convenience, we work on the communication graph $G=(V, E, \gamma)$ instead of the wireless network $M(N, A)$. 
Algorithm Dist_Implement $(\boldsymbol{V}, \boldsymbol{E}, \boldsymbol{D}, \boldsymbol{\gamma}())$

begin

1. For each node $v V \in \mathbb{D}$ do

2. Construct a single source shortest path tree $T_{v}$ in $G$ rooted at $v$;

3.Prune those branches from $T_{v}$ that do not contain nodes in $D$, and denote by $T_{v}$ the resulting tree if no confusion arises.

4. Compute the weighted sum of the edges in $T_{v}$ and store it at

endfor;

5. Find a tree $T_{v 0}$ rooted at $v 0 \in D$ from the $k=|D|$ trees such that the weighted sum of the edges in $T_{v 0}$ is the minimum. Denote by $T_{a p p}$ as $T_{v 0}$.

Let $N_{\text {Tapp }}(v)$ be the set of neighboring nodes of $v$ in $T_{a p p}$.

6. Set the power level of each node $v$ in $T_{a p p}$ by assigning its transmission power

to be $\max u \in N_{\text {Tapp }}(v)\left\{d^{2}{ }_{u, v}\right\}$.

end.

Fig. 3: A distributed algorithm Dist_Implement[4].

The total energy consumption of realizing an all-toall multicast session can be achieved if an exclusive routing tree rooted at each terminal node is used to multicast its message to the other terminal nodes. Since finding such an optimal multicast tree is complicated due to node mobility, instead, a shortest path tree rooted at each terminal node and spanning the other terminal nodes will be used. This is a multiple multicast trees based shortest path algorithm for realizing all-to-all multicast sessions[4].

\section{Performance evaluation}

In this section we evaluate the performance of the proposed algorithm against existing basic AODV. We consider wireless ad hoc networks consisting of 25, 50, 75 and 100 nodes randomly distributed in a $1000 m \times 1000 m$ region of interest. The maximum transmission range of each node is 250 meters. The fixed transmission power te is set 100 units and the reception power $r e$ is set 10 units. We assume that the maximum transmission range of each node is no more than 250 meters when its transmission power is adjustable. The length $l v$ of a message originated from a terminal node $v \in D$ is a random integer ranging from 1 to 106 . In all experiments, the value in each chart is the mean of 100 simulation results performed under 100 randomly network topologies, generated by the NS-2.29 simulator.

To evaluate the performance of the proposed algorithms, we used AODV algorithm as a performance benchmark to see how far away of the proposed solutions from this optimal one. Without loss of generality, We also used DISF and DISA to represent the corresponding versions of distributed algorithm under the models of Fixed transmission power te and Adjustable transmission power, respectively.

\subsection{Result analysis}

We finally evaluate the performance of different algorithms when the transmission power of each node is adjustable. We compute the total energy consumption of realizing an all-to-all multicast session, by the proposed approximation algorithm and distributed algorithm DISA, Fig. 4 shows number of nodes and consumed energy graph. Fig. 5 represents consumed energy and packet size graph. Fig. 6 represents consumed energy versus data rate Fig. 7 number of nodes versus packet delivery ratio Fig. 8 number of nodes versus average delay. Fig 9 represents number of nodes versus throughput. Fig. 10 shows a graph between delay versus data rate. Fig. 11 represents a graph between packet size versus delay. The network consists of 25, 50, 75 and 100 nodes, respectively. Among the algorithms, the total energy consumption by algorithm DISA is less than that by any of the other algorithms that use the single routing tree significantly. Furthermore, Fig. 4 shows that the total energy consumption by algorithm DISA is almost minimum to that by the benchmark AODV algorithm, which implies that the adoption of the shared routing tree for realizing all-to-all multicast sessions is a wise choice.

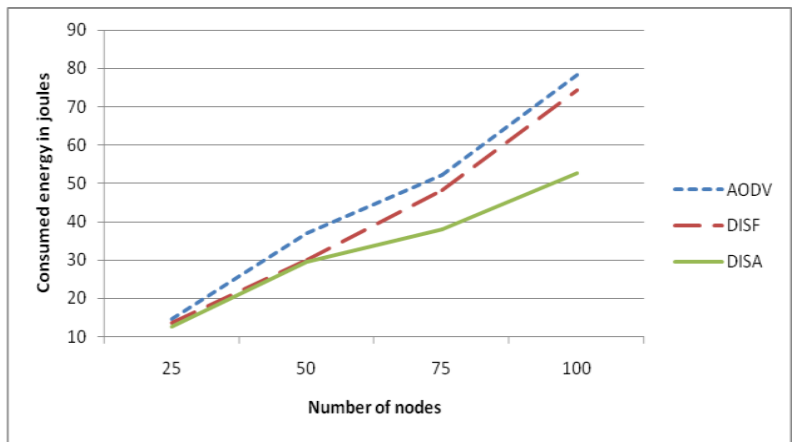

Fig. 4: Number of nodes versus consumed energy.

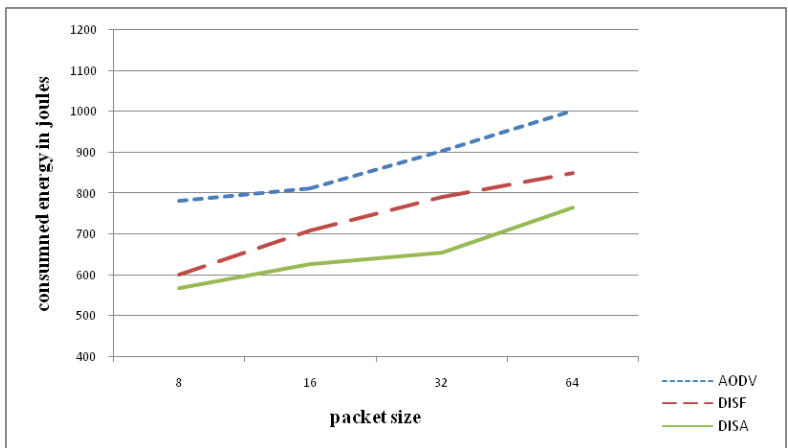

Fig. 5: Consumed energy versus packet size. 


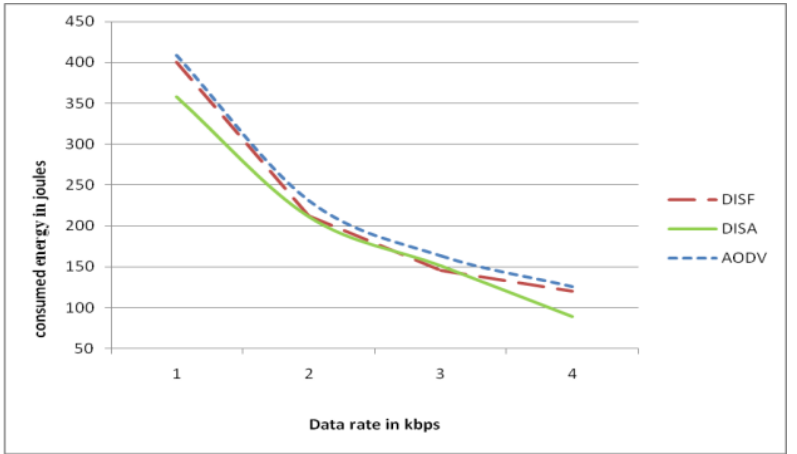

Fig. 6: Consumed energy versus data rate.

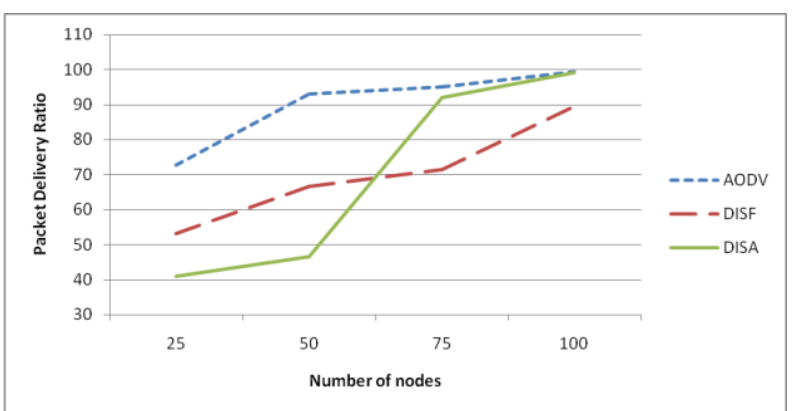

Fig. 7: Number of nodes versus packet delivery ratio.

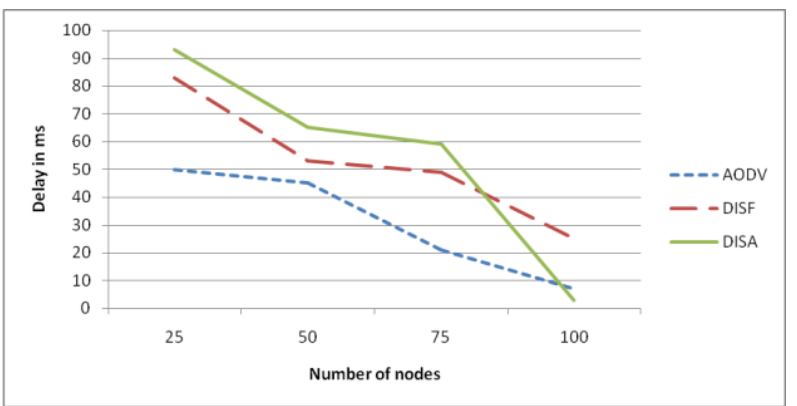

Fig. 8: Number of nodes versus delay.

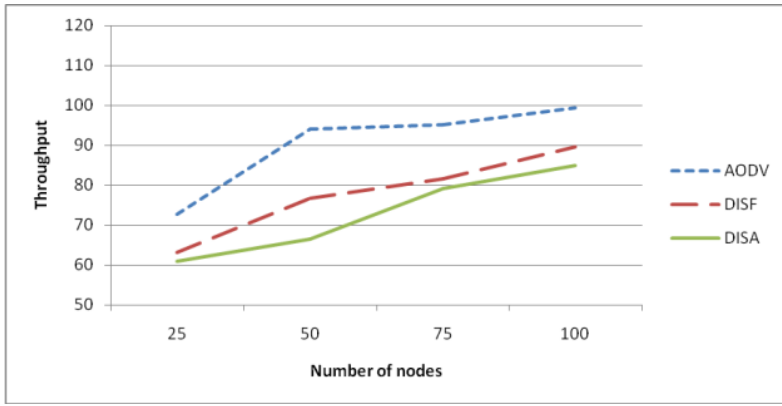

Fig. 9: Number of nodes versus throughput.

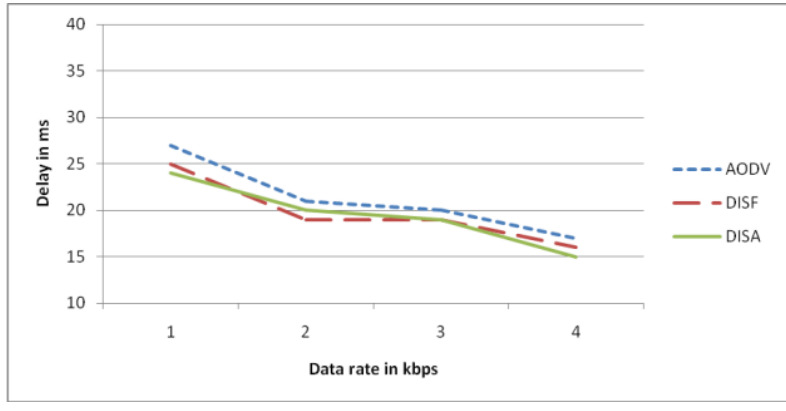

Fig. 10: Delay versus Data Rate.

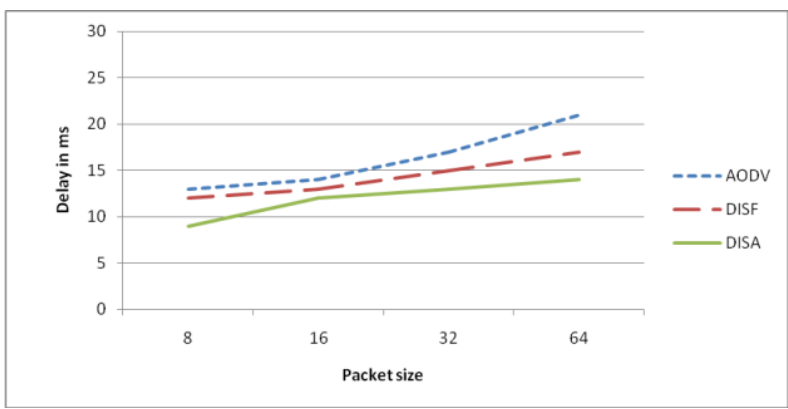

Fig. 11: Packet size versus delay.

\section{CONCLUSION}

The goal of this paper is to evaluate the performance of the distributed algorithm, when the nodes are identical and fixed It will compute the total energy consumption of an all to all multicast session with the help of distributed algorithm. The algorithm will also construct a minimum spanning tree rooted at the terminal node and spanning the all other nodes in network. The total energy consumption in the network is compared with the total number of nodes in the network.

\section{REFERENCES}

[1] Osamah S. Badarneh and Michel Kadoch "Multicast Routing Protocols in Mobile Ad Hoc Networks: A Comparative Survey and Taxonomy" EURASIP Journal on Wireless Communications and Networking Vol. 2009.

[2] V. Ramesh, Dr. P. Subbaiah N. Sandeep Chaitanya K. Sangeetha Supriya4 "An efficient energy management scheme for mobile ad-hoc networks". International Journal Of Research And Reviews In Computer Science (Ijrrcs) Vol. 1, No. 4, December 2010.

[3] Weifa Liang, Senior Member, IEEE "Approximate minimum-energy multicasting in wireless ad hoc networks" IEEE Transactions On Mobile Computing, Vol. 5, No. 4, April 2006.

[4] Weifa Liang, Senior Member, IEEE, Richard Brent, Fellow, IEEE, Yinlong $\mathrm{Xu}$, and Qingshan Wang "Minimum-energy all-to-all multicasting in wireless ad hoc networks" IEEE Transactions On Wireless Communications, Vol. 8, No. 10, October 2009.

[5] Yongwook Choi, Maleq Khan, V.S. Anil Kumar, and Gopal Pandurangan, “ Energy-optimal distributed algorithms for minimum spanning trees" IEEE Journal On Selected Areas In Communications, Vol. 27, No. 7, September 2009. 
[6] Hassan Artail, and Khaleel Mershad "MDPF: Minimum Distance Packet Forwarding for Search Applications in Mobile Ad Hoc Networks" IEEE Transactions On Mobile Computing, Vol. 8, No. 10, October 2009

[7] Maleq Khan, Gopal Pandurangan, S. Anil Kumar "Distributed Algorithms for Constructing Approximate Minimum Spanning Trees in Wireless Networks" IEEE Transactions On Parallel And Distributed Systems.

[8] Luo Junhai, Xue Liu, Ye Danxia Research on "Multicast Routing Protocols For Mobile Ad-Hoc Networks".

[9] Yongwook Choi, Maleq Khan, V.S. Anil Kumar, and Gopal Pandurangan, "Energy-Optimal Distributed
Algorithms for Minimum Spanning Trees" IEEE Journal On Selected Areas In Communications, Vol. 27, No. 7, September 2009.

[10] S.Preethi, B. Ramachandran. "Energy Efficient Routing Protocols for Mobile Ad Hoc Networks".

[11] Zhang Yi, Feng Li, Chen Wei, Zhu Lina, "PowerAware Routing Algorithm based on Mobile Agents (PARAMA) in Mobile Ad Hoc Networks" The 2008 International Conference on Embedded Software and Systems Symposia (ICESS2008). 